ISSN 1936-5098

CAE Working Paper \#10-06

\title{
On the Nature of Suppes-Sen Choice Functions
}

in an Aggregative Growth Model

by

Ram Sewak Dubey

and

Tapan Mitra

September 2010 


\title{
On the Nature of Suppes-Sen Choice Functions in an Aggregative Growth Model
}

\author{
Ram Sewak Dubey*and Tapan Mitra ${ }^{\dagger}$
}

\begin{abstract}
This paper investigates the nature of paths in the standard neoclassical aggregative model of economic growth that are maximal according to the Suppes-Sen grading principle. This is accomplished by relating such paths to paths which are utilitarian maximal when an increasing (but not necessarily concave) utility function evaluates each period's consumption. An example is presented in which an explicit form of a consumption function is described, which generates only Suppes-Sen maximal paths. This consumption function is shown to generate consumption cycles, and violate the Pigou-Dalton transfer principle.

Journal of Economic Literature Classification Numbers: D60, D70, D90.

Keywords and Phrases: Suppes-Sen Grading Principle, Utilitarian Maximality, Non-concave utility function, Consumption Cycles, PigouDalton Transfer Principle.
\end{abstract}

\footnotetext{
${ }^{*}$ Department of Economics, Cornell University, Ithaca, NY 14853; E-mail: rsd28@cornell.edu

${ }^{\dagger}$ Department of Economics, Cornell University, Ithaca, NY 14853; E-mail: tm19@cornell.edu
} 


\section{Introduction}

In a recent contribution to intertemporal social choice theory, Asheim, Bossert, Sprumont and Suzumura (2010) have investigated the nature of infinitehorizon choice functions in an aggregative productive model of economic growth. An interesting result obtained by them in this framework is that for the class of efficient paths, the choices according to the Suppes-Sen grading principle which embodies the notion of procedural equity coincide with the choices according to the Pigou-Dalton transfer principle which embodies the notion of consequentialist equity, and that both notions lead to the choice of paths which are monotone non-decreasing in consumption over time.

In this paper, we investigate the robustness of this finding by examining the equivalence result in the more standard aggregative model of economic growth, which allows for a golden-rule steady-state. More generally, this paper investigates the nature of paths (in the standard aggregative model of growth) that are maximal according to the Suppes-Sen grading principle. ${ }^{1}$

The Suppes-Sen grading principle ${ }^{2}$ is a subrelation to every social welfare quasi-ordering which respects two widely accepted guiding principles of intertemporal social choice: finite anonymity (the equal treatment of all generations) and efficiency (the positive sensitivity of the social preference structure to the well-being of each generation). Thus, a characterization of paths which are maximal according to the grading principle would be useful in making intertemporal social choices.

In the standard aggregative model of economic growth, which allows for a golden-rule steady-state, paths which are intertemporally efficient (in terms of their consumption streams) have been characterized in a classic paper by Cass (1972). When an increasing and strictly concave felicity function evaluates the period consumption, paths which are (catching-up) optimal (in terms of these felicities) have been characterized by Gale (1967). These provide (respectively) the characterization of paths which are maximal according to the efficiency quasi-ordering, and the catching-up quasi-ordering.

\footnotetext{
${ }^{1}$ For the purpose of this paper, we will interpret the grading principle as providing certain comparisons between consumption streams, rather than welfare streams, with welfare being derived from consumption, using a period welfare function. Since this is the interpretation used by Asheim, Bossert, Sprumont and Suzumura (2010), this facilitates comparison of our work with theirs.

${ }^{2}$ The grading principle is due to Suppes (1966). For a comprehensive analysis of it, see Sen (1971). Svensson (1980) provides a formal definition of the Suppes-Sen grading principle in the context of infinite utility streams. It can be characterized as the least restrictive SWR satisfying the Pareto and Anonymity axioms; see d'Aspremont (1985) and Asheim, Buchholz and Tungodden (2001).
} 
Paths which are maximal according to the grading principle fall in between the two classes mentioned above. Any path which is maximal according to the grading principle is also maximal according to the efficiency quasi-ordering. And, any path which is maximal according to the catchingup quasi-ordering is also maximal according to the grading principle.

Generalizing this last fact, a key observation ${ }^{3}$ that we use (closely related to a characterization result due to Hardy, Littlewood and Polya (1952)) is that if a path is maximal for the quasi-ordering associated with the notion of weak-maximality of Brock (1970), with an increasing felicity function evaluating the period consumption ${ }^{4}$, then it is also Suppes-Sen maximal ${ }^{5}$. This observation allows us to bring our study under the rubric of "optimal economic growth" and provides us with a convenient sufficient condition for identifying Suppes-Sen maximal paths.

It is important to caution the reader at this point that much of the theory of optimal economic growth is based on the assumption that a concave (indeed strictly concave) felicity function evaluates the period consumption. Since concavity of the felicity function plays no role in the key observation contained in the previous paragraph, a serious study of Suppes-Sen maximal paths provides a clear-cut motivation for studying optimal growth without the concavity restriction on the felicity function.

As soon as one allows the class of felicity functions to exhibit nonconcavities, a primary reason for preferring to smooth out consumption over time disappears, and optimal growth, even in the case where future felicities are not discounted ${ }^{6}$, can be compatible with cyclical behavior of consumption over time. This, in turn, suggests strongly that Suppes-Sen maximal paths need not be monotonic in our framework, and may in fact exhibit consumption cycles.

Much of the discussion in the previous paragraph is only suggestive and heuristic. A primary contribution of this paper is, therefore, to establish rigorously in an example ${ }^{7}$ of the aggregative neoclassical model that this

\footnotetext{
${ }^{3}$ This observation is straightforward for increasing felicity functions. But, for reasons that will be clear in Section 4, we define the class of felicity functions somewhat more broadly, and therefore need to establish this result as a key tool (see Proposition 1 in Section 3).

${ }^{4}$ If the felicity function is denoted by $w$, we call such a path w-maximal in this paper.

${ }^{5}$ We call such a path S-maximal in this paper.

${ }^{6}$ The fact that discounting future felicities can lead to regular cycles, in models of optimal growth with a strictly concave felicity function, is of course very well understood by this time.

${ }^{7}$ We use a technology specified by a piecewise linear production function, with the golden-rule at the kink.
} 
outcome can indeed arise. In fact, we do more. We show that Suppes-Sen maximality can be characterized by a consumption function, which we solve for explicitly. Further, we verify that such a consumption function generates cyclic paths that cannot be maximal according to the Pigou-Dalton transfer principle.

The paper is organized as follows. The framework is described in Section 2. The link between a Suppes-Sen maximal path and a path which is maximal for the quasi-ordering associated with the notion of weak-maximality of Brock (1970) is established in Section 3. In Section 4, we explicitly solve for a consumption function which generates only Suppes-Sen maximal paths, and show that such paths (i) can be cyclical, and (ii) violate the Pigou-Dalton transfer principle.

\section{The Framework}

\section{$2.1 \quad$ Notation}

Let $\mathbb{N}$ denote, as usual, the set of natural numbers $\{1,2,3, \ldots\}$, let $\mathbb{N}_{0}$ denote the set $\{0,1,2,3, \ldots\}$, and let $\mathbb{R}$ denote the set of real numbers.

For $c, c^{\prime} \in \mathbb{R}^{\mathbb{N}}$, we write $c^{\prime} \geq c$ if $c^{\prime}(t) \geq c(t)$ for all $t \in \mathbb{N}, c^{\prime}>c$ if $c^{\prime} \geq c$, and $c^{\prime} \neq c$, and $c^{\prime}>>c$ if $c^{\prime}(t)>c(t)$ for all $t \in \mathbb{N}$.

\subsection{Consumption Possibilities in an Aggregative Model}

We begin by describing the aggregative neoclassical model of economic growth. ${ }^{8}$ The framework is described by a function $f$, where $f$ represents the production function.

The production function, $f: \mathbb{R}_{+} \rightarrow \mathbb{R}_{+}$will be supposed to satisfy the following assumptions:

(F.1) $f(0)=0, f$ is increasing, concave and continuous on $\mathbb{R}_{+}$.

(F.2) There is $K \in(0, \infty)$, such that $f(x)>x$ for all $x \in(0, K)$, and $f(x)<x$ for all $x>K$.

We refer to $K$ as the maximum sustainable stock.

It can be shown that there exists $z \in(0, K)$ such that:

$$
f(z)-z \geq f(x)-x \text { for all } x \geq 0
$$

\footnotetext{
${ }^{8}$ Our version is more general than the one used in Basu and Mitra (2007), since we do not use any smoothness assumption on the production function.
} 
We will assume that this $z$ is unique. ${ }^{9}$ We refer $z$ as the golden rule stock and $g=f(z)-z$ as the golden-rule consumption.

A feasible path from $\kappa \geq 0$ is a sequence of capital stocks $\{k(t)\}$ satisfying:

$$
k(0)=\kappa, 0 \leq k(t+1) \leq f(k(t)) \text { for } t \in \mathbb{N}_{0}
$$

Associated with the feasible path $\{k(t)\}$ from $\kappa$ is a consumption sequence $\{c(t+1)\}$, defined by:

$$
c(t+1)=f(k(t))-k(t+1) \text { for } t \in \mathbb{N}_{0}
$$

Sometimes, to emphasize this association, we will denote a feasible path by $\{k(t), c(t+1)\}$.

It is easy to show that for every feasible path $\{k(t)\}$ from $\kappa \geq 0$, we have:

$$
k(t) \leq M(\kappa) \text { for } t \in \mathbb{N}_{0} ; c(t+1) \leq M(\kappa) \text { for } t \in \mathbb{N}_{0}
$$

where $\max \{K, \kappa\} \equiv M(\kappa)$. We will confine our discussion to feasible paths starting from initial stocks $\kappa \in[0, K] \equiv Y$. Then, since $M(\kappa)=K$, it follows that for every feasible path $\{k(t)\}$ from $k \in[0, K]$, we have:

$$
k(t) \leq K \text { for } t \in \mathbb{N}_{0} ; c(t+1) \leq K \text { for } t \in \mathbb{N}_{0}
$$

A feasible path $\left\{k^{\prime}(t)\right\}$ from $\kappa$ dominates a feasible path $\{k(t)\}$ from $\kappa$, if:

$$
c^{\prime}(t) \geq c(t) \text { for all } t \in \mathbb{N}
$$

with strict inequality holding in $(2)$ for at least some $t \in \mathbb{N}$.A feasible path $\{k(t)\}$ from $\kappa$ is called inefficient if there is a feasible path $\left\{k^{\prime}(t)\right\}$ from $\kappa$ which dominates it. It is efficient if it is not inefficient.

The infinite horizon consumption possibility set from the initial stock $\kappa$ is defined as:

$C(\kappa)=\{(c(1), c(2), \ldots .):.\{c(t+1)\}$ is a consumption sequence associated with a feasible path $\{k(t)\}$ from $\kappa\}$

\subsection{The Suppes-Sen Grading Principle}

A social welfare relation (SWR) is a binary relation on $C(\kappa)$. A social welfare quasi-ordering (SWQ) is a binary relation, $\succsim$, on $C(\kappa)$, which is reflexive

\footnotetext{
${ }^{9}$ Alternative sufficient conditions can be placed on $f$ to ensure this uniqueness, but we prefer at this point to simply assume the result itself, to keep the framework more general.
} 
and transitive. We associate with $\succsim$ its symmetric and asymmetric components in the usual way. Thus, we write $x \sim y$ when $x \succsim y$ and $y \succsim x$ both hold; and, we write $x \succ y$ when $x \succsim y$ holds, but $y \succsim x$ does not hold.

A SWR $\succsim_{A}$ is a subrelation to a SWR $\succsim_{B}$ if (a) $x, y \in C(\kappa)$ and $x \succsim_{A} y$ implies $x \succsim_{B} y$; and (b) $x, y \in X$ and $x \succ_{A} y$ implies $x \succ_{B} y$.

A permutation $\pi$ is a one-to-one map from $\mathbb{N}$ onto $\mathbb{N}$. Any $c \in C(\kappa)$ can be viewed as a map from $\mathbb{N}$ to $Y$, associating with each $t \in \mathbb{N}$ the element $c(t) \in Y$. The composite map $c \circ \pi$ is then a map from $\mathbb{N}$ to $Y$, associating with each $t \in \mathbb{N}$ an element $\pi(t)$ through the map $\pi$, and then associating the element $c(\pi(t)) \in Y$ through the map $c$. Thus, if $c$ is written as the sequence $(c(1), c(2), \ldots) \in C(\kappa)$, then $c \circ \pi$ is written as the sequence $(c(\pi(1)), c(\pi(2)), \ldots.) \in C(\kappa)$.

A finite permutation $\pi$ is a permutation, such that there is some $T \in \mathbb{N}$, with $\pi(t)=t$ for all $t>T$. The set of all finite permutations is denoted by $\mathcal{F}$.

The Suppes-Sen grading principle is a SWQ, denoted by $\succsim_{S}$, defined as follows. Given $c, c^{\prime} \in C(\kappa)$, we write $c^{\prime} \succsim_{S} c$ iff there is a permutation $\pi \in \mathcal{F}$ such that $c^{\prime} \circ \pi \geq c$.

A feasible path $\{k(t)\}$ from $\kappa$ is $S$-maximal if its associated consumption sequence $c \equiv\{c(t+1)\}$ is a maximal element of $C(\kappa)$, according to the grading principle. That is, a feasible path $\{k(t)\}$ from $\kappa$ is $S$-maximal if there is no feasible path $\left\{k^{\prime}(t)\right\}$ from $\kappa$, with associated consumption sequence $c^{\prime} \equiv\left\{c^{\prime}(t+1)\right\}$ satisfying $c^{\prime} \succ_{S} c$.

We are interested in characterizing the S-maximal feasible paths from an arbitrary $\kappa \in[0, K]$. These might be considered to represent the choice set of the consumption possibility set $C(\kappa)$, using the preference quasi-order $\succsim_{S}$.

\subsection{A Preliminary Result}

In attempting a characterization of the S-maximal feasible paths, considerable simplification can be obtained by noting a preliminary result, regarding the substitution possibilities of the consumption possibility set. Specifically, given a feasible path $\{k(t)\}$ with positive consumption in some period $T$, it is possible to construct another feasible path which has a bit less consumption in period $T$, but more consumption in all other periods, compared to the consumption sequence on the path $\{k(t)\}$.

Lemma 1 Let $\{k(t)\}$ be a feasible path from $\kappa \in[0, K]$, with associated consumption sequence $\{c(t+1)\}$. Let $T \in \mathbb{N}$, and let $0 \leq \gamma<c(T)$. Then, 
there is a feasible path $\left\{k^{\prime}(t)\right\}$ from $\kappa$, with associated consumption sequence $\left\{c^{\prime}(t+1)\right\}$, such that $c^{\prime}(T)>\gamma$, and $c^{\prime}(t+1)>c(t+1)$ for all $t \neq T-1$.

The Lemma yields the useful corollary that if a feasible path $\{k(t)\}$ is not S-maximal, then there is a feasible path $\left\{k^{\prime}(t)\right\}$ and a finite permutation such that the permuted consumption sequence on $\left\{k^{\prime}(t)\right\}$ exceeds the consumption sequence on $\{k(t)\}$ in all periods.

Corollary 1 Let $c \in C(\kappa)$, and suppose $c$ is not $S$-maximal. Then, there is $\bar{c} \in C(\kappa)$ and $\pi \in \mathcal{F}$, such that:

$$
\bar{c}(\pi(t))>c(t) \text { for all } t \geq 1
$$

\section{The Grading Principle and Utilitarianism}

Permutations involved in the definition of the grading principle make the study of S-maximal paths technically difficult. It becomes cumbersome to look for permutations under which two consumption streams become comparable according to the quasi-ordering.

This difficulty can be circumvented by relating the grading principle to a utilitarian relation (for a class of felicity functions), so that consumption streams which are maximal according to the utilitarian relation yield consumption streams which are S-maximal. Since the utilitarian relation has been studied extensively in ranking consumption streams in growth models, establishing the connection has the potential of making familiar techniques (which, incidentally, do not involve permutations) available to us for the study of S-maximal paths. This is the approach developed in this section, and applied to a concrete example in the next section, demonstrating that it is indeed a fruitful detour.

The result connecting the grading principle and utilitarianism (in comparing finite streams) is directly obtained from a classic characterization reported by Hardy, Littlewood and Polya (1952), in their definitive study on mathematical inequalities.

Lemma 2 (Hardy, Littlewood and Polya) Let $(x(1), \ldots, x(n)),\left(x^{\prime}(1), \ldots, x^{\prime}(n)\right)$ be vectors in $\mathbb{R}^{n}$.

(i) If, for a permutation $\pi$ of the integers $(1, \ldots, n)$, we have $\left(x^{\prime}(\pi(1)), \ldots, x^{\prime}(\pi(n))\right) \geq$ $(x(1), \ldots, x(n))$, then for every increasing and continuous function $g: \mathbb{R} \rightarrow \mathbb{R}$,

$$
\sum_{i=1}^{n} g\left(x^{\prime}(i)\right) \geq \sum_{i=1}^{n} g(x(i))
$$


(ii) If for every increasing and continuous function $g: \mathbb{R} \rightarrow \mathbb{R}$,

$$
\sum_{i=1}^{n} g\left(x^{\prime}(i)\right) \geq \sum_{i=1}^{n} g(x(i))
$$

then there is a permutation $\pi$ of the integers $(1, \ldots, n)$, such that $\left(x^{\prime}(\pi(1)), \ldots, x^{\prime}(\pi(n))\right) \geq$ $(x(1), \ldots, x(n))$.

In contrast to Lemma 2, we will be concerned with comparison of infinite streams. The grading principle still involves permutation of a finite number of elements of the stream (although this finite number is not fixed in advance) and therefore does not create additional problems. However, one will have to choose the utilitarian criterion more carefully, since the sums in Lemma 2 will typically not be well-defined for infinite streams.

Another difference is that we will want the utilitarian comparisons for a somewhat broader class of felicity functions than are allowed in Lemma 2 (for reasons which can best be appreciated when the theory is applied to a concrete example in Section 4). We take up each of these issues in the following subsections.

\subsection{A Class of Felicity Functions}

Let us denote by $W_{N D}$ the class of non-decreasing functions from $\mathbb{R}_{+}$to $\mathbb{R}$. We define the subclass $W$ of $W_{N D}$ by:

$$
W=\left\{w \in W_{N D}: \text { there exists } \bar{c} \in \mathbb{R}_{+} \text {, such that } w(c)>w(\bar{c}) \text { for all } c>\bar{c}\right\}
$$

Clearly $W$ includes the class of all increasing functions from $\mathbb{R}_{+}$to $\mathbb{R}$, and it includes the class of non-decreasing functions which are increasing beyond a certain point in its domain.

For $w \in W$, we define:

$$
D(w)=\left\{\bar{c} \in \mathbb{R}_{+}: w(c)>w(\bar{c}) \text { for all } c>\bar{c}\right\}
$$

These are points $(\bar{c})$ in the domain of $w$, such that increasing $c$ will lead to a higher felicity than at $\bar{c}$.

\subsection{Utilitarian Maximality}

The utilitarian social welfare relation that we use is based on the notion of weak-maximality used by Brock (1970) in the optimal growth literature. 
For $c, c^{\prime} \in C(\kappa)$, and $w \in W$, we write $c^{\prime} \succsim_{w} c$ iff:

$$
\lim \inf _{T \rightarrow \infty} \sum_{t=1}^{T}\left[w(c(t))-w\left(c^{\prime}(t)\right)\right] \leq 0
$$

A basic property of the social welfare relation can be stated as follows.

Lemma 3 For $c, c^{\prime} \in C(\kappa)$, and $w \in W$, we have $c^{\prime} \succ_{w} c$ if and only if:

$$
\lim \inf _{T \rightarrow \infty} \sum_{t=1}^{T}\left[w\left(c^{\prime}(t)\right)-w(c(t))\right]>0
$$

Let $\bar{c} \in C(\kappa)$ and $w \in W$. Then $\bar{c}$ is called $w$-maximal if there is no $c \in C(\kappa)$ satisfying $c \succ_{w} \bar{c}$. Then, by Lemma 3 , we see that given $\bar{c} \in C(\kappa)$ and $w \in W, \bar{c}$ is $w$-maximal if for every $c \in C(\kappa)$, we have:

$$
\lim \inf _{T \rightarrow \infty} \sum_{t=1}^{T}[w(c(t))-w(\bar{c}(t))] \leq 0
$$

\subsection{Utilitarian Maximality implies Suppes-Sen Maximality}

We are now in a position to state the main result of this section. If for some $w \in W$, we know that $\bar{c} \in C(\kappa)$ is w-maximal, then we can conclude that $\bar{c}$ is also S-maximal. This gives us a useful sufficient condition for obtaining S-maximal paths (which does not involve permutations). It is particularly oriented to applications because we have chosen the class $W$ to be very broad, and we only need to check w-maximality for a single element $w$ of this class.

Proposition 1 Let $\bar{c} \in C(\kappa)$, and suppose that for some $w \in W$, with $\bar{c}(N) \in D(w)$ for some $N \in \mathbb{N}$, it is w-maximal. Then, it is also $S$-maximal.

\section{Suppes-Sen Maximality and Consumption Cy- cles}

The purpose of this section is to demonstrate that Suppes-Sen maximality can lead to the choice of a growth path which is cyclical in consumption. In fact, we show this in a particularly compelling way by obtaining explicitly a consumption function, which (a) generates only Suppes-Sen maximal paths, 
and (b) all such Suppes-Sen maximal paths exhibit period-two consumption cycles after at most a finite number of time periods.

The consumption function is derived by solving for w-maximal paths for a particular choice of $w \in W$, thereby showing the usefulness of the results of Section 3. The nature of the consumption function is such that it leads to consumption cycles, and this aspect can be best understood by recognizing that this is to be expected if we solve for w-maximal paths for a choice of $w \in W$, which is suitably non-concave.

It is useful to point out that studying w-maximality with a non-concave $w \in W$ is not the objective of this study. It is only a device which enables us to obtain an S-maximal consumption function (by relying on Proposition 1, which establishes the connection between the two concepts). However, it is also useful to recognize that the study of S-maximality leads very naturally to the study of w-maximality with $w \in W$ which are not necessarily concave.

Studying w-maximality with a $w \in W$ which is not concave is, of course, far from straightforward. Almost all the literature on (undiscounted) optimal growth deals with w-maximality (or one of its variants) for $w \in W$, which is also concave (mostly, strictly concave). However, as this section shows, the available techniques can be suitably modified to study w-maximality with the specific non-concave $w \in W$ that we use. We hope that these methods can be used in other contexts which involve dynamic optimization over an infinite horizon with a not necessarily concave return function.

\subsection{S-Maximal Consumption Function}

A consumption function is a rule $h:[0, K] \rightarrow[0, K]$, assigning a consumption choice $h(y) \in[0, K]$ for every output level $y \in[0, K]$, such that $h(y) \leq y$.

Note that a consumption function is time-independent. Also, the consumption choice $h(y)$ depends only on the output level $y$, and not on aspects like how and when the output level $y$ is attained. It says that, whenever the output level is $y$, the consumption choice is $h(y)$.

A consumption function $h$ generates a feasible path $\{k(t)\}$ starting from any $\kappa \in[0, K]$ as follows:

$$
k(0)=\kappa, k(t+1)=f(k(t))-h(f(k(t))) \text { for all } t \in \mathbb{N}_{0}
$$

Its associated consumption sequence $\{c(t+1)\}$ is given by:

$$
c(t+1)=h(f(k(t))) \text { for all } t \in \mathbb{N}_{0}
$$

An $S$-maximal consumption function is a consumption function, $h$, such 
that for every $\kappa \in[0, K]$, the consumption sequence associated with the feasible path generated by $h$ is S-maximal in $C(\kappa)$.

\subsection{An Example}

We now analyze in detail a specific example of the framework described in Section 2, in the context of which we solve for an S-maximal consumption function.

\subsubsection{Specification of Consumption Possibilities}

The production function, $f$, is piecewise linear and specified by two parameters $(a, b)$, with $a>1$ and $a b=1$. For a numerical example, consider $a=2$ and $b=(1 / 2)$. The function, $f$, is defined as follows:

$$
f(x)= \begin{cases}a x & \text { for } x \in[0,1] \\ a+b(x-1) & \text { for } x>1\end{cases}
$$

The golden-rule stock, $z$, is equal to 1 , and the maximum sustainable stock, $K$, is $[(a-b) /(1-b)]$. The golden-rule consumption is $(a-1)$.

Define the transition possibility set, $\Omega$, by:

$$
\Omega=\left\{\left(x, x^{\prime}\right) \in \mathbb{R}_{+}^{2}: x^{\prime} \leq f(x)\right\}
$$

and the two-period transition possibility set, $\Lambda$, by:

$\Lambda=\left\{\left(x, x^{\prime \prime}\right) \in \mathbb{R}_{+}^{2}:\right.$ there is $x^{\prime} \in \mathbb{R}_{+}$, such that $\left(x, x^{\prime}\right) \in \Omega$ and $\left.\left(x^{\prime}, x^{\prime \prime}\right) \in \Omega\right\}$

For $\left(x, x^{\prime \prime}\right) \in \Lambda$, we also define $\Gamma\left(x, x^{\prime \prime}\right)=\left\{x^{\prime} \in \mathbb{R}_{+}:\left(x, x^{\prime}\right) \in \Omega\right.$ and $\left.\left(x^{\prime}, x^{\prime \prime}\right) \in \Omega\right\}$.

\subsubsection{Specification of Felicity Function}

Denote $(a-b)$ by $\theta$, and define $w$ by:

$$
w(c)= \begin{cases}0 & \text { for } c \in[0, \theta) \\ c & \text { for } c \geq \theta\end{cases}
$$

Clearly, $w$ is not concave, not increasing (on $[0, \theta)$ ) and not continuous (at $\theta)$. However, note that $w \in W$, and $D(w)=[\theta, \infty)$. [We defined the class $W$ to be broad enough to accommodate such felicity functions]. A useful property of $w$ is that $w(c) \leq c$ for all $c \geq 0$.

Given the felicity function $w$, we define a reduced-form utility function $u: \Omega \rightarrow \mathbb{R}$ by:

$$
u\left(x, x^{\prime}\right)=w\left(f(x)-x^{\prime}\right) \text { for all }\left(x, x^{\prime}\right) \in \Omega
$$




\subsubsection{Price Support of a Period Two Cycle}

It should be clear that, with the chosen felicity function $w$, the golden-rule loses its traditional importance in optimal growth models. Staying at the golden-rule forever cannot be w-maximal. Since the golden rule consumption is $(a-1)<(a-b) \equiv \theta$, and so $w(a-1)=0$, the felicity sequence along the golden-rule path is $\{0,0,0, \ldots\}$, while the feasible path $(1, b, 1, b, \ldots)$ from the golden-rule stock yields the felicity sequence $(\theta, 0, \theta, 0, \ldots$.$) .$

The central importance of the golden-rule and its price support is usurped by a specific period two cycle and its price support. This makes the traditional methods still applicable by viewing the period two cycle as stationary in terms of the two-period transition possibility set, $\Lambda$.

The specific cycle of interest is specified by the stock sequence $(b, 1, b, 1, \ldots)$, and associated consumption sequence $(0, \theta, 0, \theta, \ldots$.$) . The stationary price$ support for it is $p=1$. The following Lemma, summarizing this result, is crucial to much of the subsequent analysis.

Lemma 4 Let $\left(x, x^{\prime}\right) \in \Omega$, and $\left(x^{\prime}, x^{\prime \prime}\right) \in \Omega$. Then,

$$
u\left(x, x^{\prime}\right)+u\left(x^{\prime}, x^{\prime \prime}\right)+x^{\prime \prime}-x \leq \theta=u(b, 1)+u(1, b)
$$

\subsubsection{Value Losses}

In the standard theory of optimal intertemporal allocation, when future utilities are not discounted, and the felicity function is increasing and strictly concave, the nature of (catching-up) optimal paths can be examined by focusing on value losses suffered by a path for not operating at the goldenrule.

In our context, given the price support of the period two cycle in Lemma 4 , it is natural to focus instead on value losses suffered by a path for not operating at the period-two cycle, and this is precisely what we do.

For $\left(x, x^{\prime \prime}\right) \in \Lambda$, we can define:

$$
R\left(x, x^{\prime \prime}\right)=\sup _{x^{\prime} \in \Gamma\left(x, x^{\prime \prime}\right)}\left\{u\left(x, x^{\prime}\right)+u\left(x^{\prime}, x^{\prime \prime}\right)+x^{\prime \prime}-x\right\}
$$

By Lemma 4 , we know that $R\left(x, x^{\prime \prime}\right) \leq \theta$ for all $\left(x, x^{\prime \prime}\right) \in \Lambda$. We define the value-loss from operating at $\left(x, x^{\prime \prime}\right) \in \Lambda$ by:

$$
L\left(x, x^{\prime \prime}\right)=\theta-R\left(x, x^{\prime \prime}\right)
$$

Clearly, $L\left(x, x^{\prime \prime}\right) \geq 0$ for all $\left(x, x^{\prime \prime}\right) \in \Lambda$. 
We now establish a version of the value-loss lemma of Radner (1961), Atsumi (1965) and McKenzie (1968) for our framework. This ensures that being uniformly away from the period-two cycle produces a uniform value loss.

Lemma 5 Given any $\alpha \in(0, b]$, there is $\beta>0$, such that whenever $\left(x, x^{\prime \prime}\right) \in$ $\Lambda$, and $(b-x) \geq \alpha$, we have:

$$
L\left(x, x^{\prime \prime}\right) \geq \beta
$$

\subsubsection{S-maximal Consumption Function}

The price-support property of the period two cycle in Lemma 4, and the value-loss property of Lemma 5 enable us to use suitable modifications of standard arguments in the theory of undiscounted (catching-up) optimal growth and show that the consumption function, $h:[0, K] \rightarrow[0, K]$, defined by:

$$
h(y)= \begin{cases}0 & \text { for } y \in[0, a) \\ y-b & \text { for } y \in[a, K]\end{cases}
$$

is a $w$-maximal consumption function for the $w$ specified in $(\mathrm{W})$, and therefore an S-maximal consumption function (by Proposition 1).

The consumption function $h$ leads to the following dynamical system, governing the behavior over time of the capital stock sequence $\{k(t)\}$ generated by it, starting from any initial stock $\kappa \in[0, K]$ :

$$
k(t+1)=\left\{\begin{array}{ll}
a k(t) & \text { for } k(t) \in[0,1) \\
b & \text { for } k(t) \in[1, K]
\end{array} ; k(0)=\kappa\right.
$$

Thus, starting from any initial stock $\kappa \in(0, K]$, the capital stock sequence $\{k(t)\}$ generated by the S-maximal consumption function $h$ coincides with the period two cycle $\{b, 1, b, 1, .$.$\} after at most a finite number of periods.$ This means that after at most a finite number of periods, the feasible path generated by the S-maximal consumption function $h$ exhibits the consumption cycle $\{0, \theta, 0, \theta, .$.$\} .$

We summarize these findings in the following theorem.

Theorem 1 (A)The consumption function $h$, specified in $(C F)$, is (a) a $w$-maximal consumption function for the $w$ specified in $(W)$, and (b) an $S$-maximal consumption function. That is, every path generated by $h$ is a Suppes-Sen maximal path.

(B) Every path from $\kappa \in(0, K]$, generated by the consumption function $h$, exhibits period-two consumption cycles after at most a finite number of time periods. 


\subsubsection{Pigou-Dalton Transfer Principle}

Following Asheim, Bossert, Sprumont and Suzumura (2010), we will say that a consumption function $h$ satisfies the Pigou-Dalton transfer principle if for all $\kappa \in[0, K]$, and for all non-negative sequences $\{c(t+1)\}_{0}^{\infty},\left\{c^{\prime}(t+1)\right\}_{0}^{\infty}$, (i) if there exist $\varepsilon>0$ and $S, T \in \mathbb{N}_{0}$ such that $c(S+1)=c^{\prime}(S+1)-\varepsilon \geq c^{\prime}(T+$ 1) $+\varepsilon=c(T+1)$, and $c(t+1)=c^{\prime}(t+1)$ for all $t \in \mathbb{N}_{0} /\{S, T\}$, and further (ii) $\left\{c^{\prime}(t+1)\right\}_{0}^{\infty}$ is the consumption sequence generated by the consumption function $h$ starting with the initial capital stock $\kappa$, then $\{c(t+1)\}_{0}^{\infty}$ cannot be a consumption sequence associated with a feasible path starting from $\kappa$.

We now show that for the production function $f$ given by $(\mathrm{PF})$, the consumption function $h$ given by (CF) does not satisfy the Pigou-Dalton transfer principle.

Consider the feasible path $\left\{k^{\prime}(t)\right\}$ from $\kappa=1$, defined by the sequence of capital stocks $\{1, b, 1, b, \ldots\}$, with associated consumption sequence $\left\{c^{\prime}(t+1)\right\}$ given by $\{\theta, 0, \theta, 0, \ldots\}$. It is easy to check that $\left\{c^{\prime}(t+1)\right\}$ is the consumption sequence generated by the consumption function $h$ starting with the initial capital stock $\kappa=1$.

Define the sequence $\{k(t)\}$ from $\kappa=1$ as follows:

$$
\left.\begin{array}{l}
k(0)=k=1 \\
k(1)=b+\varepsilon \\
k(2)=1+(a-1) \varepsilon \\
k(t+1)=f(k(t))-c^{\prime}(t+1) \quad \text { for all } t \geq 2
\end{array}\right\}
$$

where $0<\varepsilon<(a-b) / 2$. Clearly $k(1)>k^{\prime}(1)$ and $k(2)>k^{\prime}(2)$. It is now easy to verify recursively from the last line of (9) that $k(t)>k^{\prime}(t)$ for all $t>2$. Further, since $f(k(0))=f(1)=a$, and $k(1)=b+\varepsilon$, we have $f(k(0))-k(1)=(a-b)-\varepsilon=c^{\prime}(1)-\varepsilon>0$. And since $k(1)<b+[(a-b) / 2]<a$, we have $f(k(1))-k(2)=(1+a \varepsilon)-[1+(a-1) \varepsilon]=\varepsilon=c^{\prime}(2)+\varepsilon>0$.

Thus, using the last line of $(9)$, it follows that $\{k(t)\}$ is a feasible path from $\kappa=1$, with associated consumption sequence $\{c(t+1)\}$ given by $c(1)=$ $c^{\prime}(1)-\varepsilon, c(2)=c^{\prime}(2)+\varepsilon$, and $c(t+1)=c^{\prime}(t+1)$ for all $t \geq 2$. By choice of $\varepsilon$, we have:

$$
c(1)=c^{\prime}(1)-\varepsilon=(a-b)-\varepsilon>\varepsilon=c^{\prime}(2)+\varepsilon=c(2)
$$

Thus, $h$ violates the Pigou-Dalton transfer principle, by using $S=0$ and $T=1$. 


\section{Appendix: Proofs}

Proof of Lemma 1. Let $T=1$. Since $f(0)=0$, and $c(1)>0$, we have $k(0)>0$. Since $f$ is continuous, we can pick $\varepsilon(1)>0$ (sufficiently close to 0 ) to ensure that $\varepsilon(1)<c(1)-\gamma$. For $t \geq 1$, define iteratively:

$$
\varepsilon(t+1)=(1 / 2)[f(k(t)+\varepsilon(t))-f(k(t)]
$$

Then, $\{\varepsilon(t)\}$ is well-defined, and $\varepsilon(t)>0$ for $t \geq 1$, since $f$ is increasing.

Now define $k^{\prime}(0)=k(0)$, and $k^{\prime}(t)=k(t)+\varepsilon(t)$ for $t \geq 1$. Then,

$$
\begin{aligned}
f(k(0))-k^{\prime}(1) & =f(k(0))-k(1)-\varepsilon(1) \\
& =c(1)-\varepsilon(1)>\gamma
\end{aligned}
$$

and for $t \geq 1$,

$$
\begin{aligned}
f\left(k^{\prime}(t)\right)-k^{\prime}(t+1) & =f(k(t)+\varepsilon(t))-k(t+1)-\varepsilon(t+1) \\
& =f(k(t)+\varepsilon(t))-f(k(t))+f(k(t))-k(t+1)-\varepsilon(t+1) \\
& =c(t+1)+\varepsilon(t+1)
\end{aligned}
$$

Clearly, (10) and (11) establish that $\left\{k^{\prime}(t)\right\}$ is a feasible path from $\kappa$, and that its associated consumption sequence $\left\{c^{\prime}(t+1)\right\}$ satisfies $c^{\prime}(1)>\gamma$, and $c^{\prime}(t+1)>c(t+1)$ for all $t \neq 0$.

The case $T>1$ can be handled similarly in the forward direction, and with suitable decreases in the stock sequence in the backward direction.

Proof of Corollary 1. Since $c$ is not S-maximal, there is $c^{\prime} \in C(\kappa)$, and $\pi \in \mathcal{F}$, such that:

$$
c^{\prime} \circ \pi>c
$$

Using (12), there is a time period, $\tau$, for which $c^{\prime}(\pi(\tau))>c(\tau)$. Denote $\pi(\tau)$ by $T$, and $c(\tau)$ by $\gamma$. Then, using Lemma 1 , there is a feasible path $\{\bar{k}(t)\}$ from $\kappa$, with associated consumption sequence $\{\bar{c}(t)\}$, such that $\bar{c}(t)>c^{\prime}(t)$ for all $t \neq T$, and $\bar{c}(T)>\gamma \equiv c(\tau)$. Then, for every $s \in \mathbb{N}$, with $s \neq \tau$, we have $\bar{c}(\pi(s))>c^{\prime}(\pi(s)) \geq c(s)$ by (12). And, for $s=\tau$, we have $\bar{c}(\pi(s))=$ $\bar{c}(T)>\gamma=c(\tau)=c(s)$. This establishes $(3)$.

Proof of Lemma 3. If $c^{\prime} \succ_{w} c$, then $c^{\prime} \succsim_{w} c$ holds and $c \succsim_{w} c^{\prime}$ does not hold. The former restriction implies that (4) holds. The latter restriction implies that the following inequality does not hold:

$$
\lim \inf _{T \rightarrow \infty} \sum_{t=1}^{T}\left[w\left(c^{\prime}(t)\right)-w(c(t))\right] \leq 0
$$


Thus, (5) must hold.

To go in the opposite direction, let $c, c^{\prime} \in C(\kappa)$, and $w \in W$, and suppose (5) holds. This means:

$$
\begin{aligned}
\lim \inf _{T \rightarrow \infty} \sum_{t=1}^{T}\left[w(c(t))-w\left(c^{\prime}(t)\right)\right] & \leq \lim \sup _{T \rightarrow \infty} \sum_{t=1}^{T}\left[w(c(t))-w\left(c^{\prime}(t)\right)\right] \\
& =-\lim \inf _{T \rightarrow \infty} \sum_{t=1}^{T}\left[w\left(c^{\prime}(t)\right)-w(c(t))\right] \\
& <0
\end{aligned}
$$

Clearly (13) means that $c^{\prime} \succsim_{w} c$, and (5) means that $c \succsim_{w} c^{\prime}$ does not hold. Thus, we have $c^{\prime} \succ_{w} c$.

Proof of Proposition 1. Suppose, on the contrary, there is some $c \in C(\kappa)$, such that $c \succ_{S} \bar{c}$. Then, there is $\pi \in \mathcal{F}$, such that $c \circ \pi>\bar{c}$. By Corollary 1 , there is $c^{\prime} \in C(\kappa)$ such that $c^{\prime} \circ \pi>>\bar{c}$. Since $\pi \in \mathcal{F}$, we can pick $N^{\prime} \in \mathbb{N}$, such that $N^{\prime} \geq N$, and $\pi(t)=t$ for all $t \geq N^{\prime}$. Then, we must have:

$$
\left(c^{\prime}(\pi(1)), \ldots, c^{\prime}\left(\pi\left(N^{\prime}\right)\right)\right)>>\left(\bar{c}(1), \ldots, \bar{c}\left(N^{\prime}\right)\right)
$$

and:

$$
c^{\prime}(t)=c^{\prime}(\pi(t))>\bar{c}(t) \text { for all } t>N^{\prime}
$$

Since $\pi(t)=t$ for all $t \geq N^{\prime}, \pi$ must map $\left\{1, \ldots, N^{\prime}\right\}$ onto $\left\{1, \ldots, N^{\prime}\right\}$. Since $w \in W, N \leq N^{\prime}$, and $\bar{c}(N) \in D(w)$, it follows from (14) that:

$$
\sum_{t=1}^{N^{\prime}} w\left(c^{\prime}(t)\right)=\sum_{t=1}^{N^{\prime}} w\left(c^{\prime}(\pi(t))>\sum_{t=1}^{N^{\prime}} w(\bar{c}(t))\right.
$$

And, since $W \subset W_{N D}$, it follows from (15) that for all $T>N^{\prime}$,

$$
\sum_{t=N^{\prime}+1}^{T} w\left(c^{\prime}(t)\right) \geq \sum_{t=N^{\prime}+1}^{T} w(\bar{c}(t))
$$

Denoting $\left[\sum_{t=1}^{N^{\prime}} w\left(c^{\prime}(t)\right)-\sum_{t=1}^{N^{\prime}} w(\bar{c}(t))\right]$ by $\alpha$, we have $\alpha>0$. Using (16), we have for all $T>N^{\prime}$ :

$$
\sum_{t=1}^{T}\left[w\left(c^{\prime}(t)\right)-w(\bar{c}(t))\right] \geq\left[\sum_{t=1}^{N^{\prime}} w\left(c^{\prime}(t)\right)-\sum_{t=1}^{N^{\prime}} w(\bar{c}(t))\right]=\alpha
$$

which contradicts the w-maximality of $\bar{c}$. 
Proof of Lemma 4. Clearly $u(b, 1)=w(0)=0$, and $u(1, b)=$ $w(a-b)=a-b \equiv \theta$. Thus, we have:

$$
\theta=u(b, 1)+u(1, b)
$$

It remains to establish the inequality:

$$
u\left(x, x^{\prime}\right)+u\left(x^{\prime}, x^{\prime \prime}\right)+x^{\prime \prime}-x \leq \theta
$$

We break up the demonstration into cases:(i) $x<b$ or $x^{\prime}<b$; (ii) $x \geq b$ and $x^{\prime} \geq b$. Clearly, we have:

$$
\begin{aligned}
u\left(x, x^{\prime}\right)+u\left(x^{\prime}, x^{\prime \prime}\right)+x^{\prime \prime}-x & \leq f(x)-x^{\prime}+f\left(x^{\prime}\right)-x^{\prime \prime}+x^{\prime \prime}-x \\
& =[f(x)-x]+\left[f\left(x^{\prime}\right)-x^{\prime}\right]
\end{aligned}
$$

Thus, if $x<b$, then $f(x)-x<f(b)-b$ (since $b<z=1)=1-b$, while $f\left(x^{\prime}\right)-x^{\prime} \leq f(z)-z=a-1$, so that:

$$
[f(x)-x]+\left[f\left(x^{\prime}\right)-x^{\prime}\right]<(1-b)+(a-1)=(a-b)
$$

Clearly, (19) also holds if $x^{\prime}<b$. Thus, (17) always holds in case (i).

We subdivide case (ii) into two subcases: (A) $f(x)-x^{\prime}<\theta$, (B) $f(x)-$ $x^{\prime} \geq \theta$. In subcase $(\mathrm{A})$, we have:

$$
\begin{aligned}
u\left(x, x^{\prime}\right)+u\left(x^{\prime}, x^{\prime \prime}\right)+x^{\prime \prime}-x & =u\left(x^{\prime}, x^{\prime \prime}\right)+x^{\prime \prime}-x \leq f\left(x^{\prime}\right)-x^{\prime \prime}+x^{\prime \prime}-x \\
& =f\left(x^{\prime}\right)-x \leq f(f(x))-x \\
& =f(f(x))-f(1)+f(1)-x \\
& \leq f_{+}^{\prime}(1)[f(x)-1]+a-x=b f(x)-x+(a-b) \\
& \leq \text { bax }-x+(a-b)=(a-b)
\end{aligned}
$$

which establishes (17).

In subcase (B), we have $f(x) \geq(a-b)+x^{\prime} \geq(a-b)+b=a$, and so $x \geq 1$. Let $(x-1)=\varepsilon$; then $\varepsilon \geq 0$. Also, define $f(x)-x^{\prime}-\theta$ by $\delta$; then $\delta \geq 0$. Then $f(x)=f(1+\varepsilon)=a+b \varepsilon$, and so:

$$
\delta=f(1+\varepsilon)-x^{\prime}-\theta=a+b \varepsilon-x^{\prime}-(a-b)=b(1+\varepsilon)-x^{\prime}
$$

This means:

$$
x^{\prime}=b(1+\varepsilon)-\delta
$$

and:

$$
f\left(x^{\prime}\right)-x \leq a x^{\prime}-x=a b(1+\varepsilon)-a \delta-x=-a \delta
$$


Using (21), we obtain:

$$
\begin{aligned}
u\left(x, x^{\prime}\right)+u\left(x^{\prime}, x^{\prime \prime}\right)+x^{\prime \prime}-x & \leq f(x)-x^{\prime}+f\left(x^{\prime}\right)-x^{\prime \prime}+x^{\prime \prime}-x \\
& =f(x)-x^{\prime}+f\left(x^{\prime}\right)-x \\
& =\theta+\delta-a \delta \leq \theta
\end{aligned}
$$

which again establishes (17).

Proof of Lemma 5. Define $\beta=[f(b)-b]-[f(b-\alpha)-(b-\alpha)]$. Then $\beta>0$ since $f(k)-k$ is increasing in $k$ for $k \in[0,1), b \in(0,1)$ and $\alpha \in(0, b]$.

Suppose, contrary to (8), there is $\left(x, x^{\prime \prime}\right) \in \Lambda$, such that $L\left(x, x^{\prime \prime}\right)<\beta$. Then, $R\left(x, x^{\prime \prime}\right)>\theta-\beta$, and by definition of $R$, there is some $x^{\prime} \in \Gamma\left(x, x^{\prime \prime}\right)$, such that:

$$
u\left(x, x^{\prime}\right)+u\left(x^{\prime}, x^{\prime \prime}\right)+x^{\prime \prime}-x>\theta-\beta
$$

However,

$$
\begin{aligned}
u\left(x, x^{\prime}\right)+u\left(x^{\prime}, x^{\prime \prime}\right)+x^{\prime \prime}-x & \leq f(x)-x^{\prime}+f\left(x^{\prime}\right)-x^{\prime \prime}+x^{\prime \prime}-x \\
& =f(x)-x+f\left(x^{\prime}\right)-x^{\prime} \\
& \leq f(x)-x+f(1)-1=f(x)-x+(a-1) \\
& \leq[f(b-\alpha)-(b-\alpha)]+(a-1) \\
& =[f(b)-b]-\beta+(a-1) \\
& =(1-b)-\beta+(a-1)=\theta-\beta
\end{aligned}
$$

the inequality in the fourth line of $(24)$ following from the facts that $f(k)-k$ is increasing in $k$ for $k \in[0,1)$, and $0 \leq x \leq b-\alpha<b<1$. Clearly, (24) contradicts (23), establishing (8).

Proof of Theorem 1. (A) It is sufficient to prove (a), since then (b) follows from Proposition 1. Our proof of (a) is facilitated by considering three possibilities for the initial stock: (i) $\kappa \in[b, 1$ ); (ii) $\kappa \in[1, K]$; (iii) $\kappa \in[0, b)$.

Case (i) $(\kappa \in[b, 1))$

In this case, the consumption function $h$ generates the stock sequence $\{k(t)\}=(\kappa, f(\kappa), b, 1, b, 1, \ldots)$ with associated consumption sequence $\{c(t+$ $1)\}=\left(0, f^{2}(\kappa)-b, 0, a-b, 0, a-b, \ldots\right)$.

We verify that for each $t=0,2,4, \ldots$, the value loss $L(k(t), k(t+2))=0$. For $t \in\{2,4, \ldots\}$, this follows from Lemma 4. For $t=0, L(k(t), k(t+2))=$ 
$L(\kappa, b)$. Now, $f^{2}(\kappa)-b \geq f(1)-b=a-b$, so:

$$
\begin{aligned}
R(\kappa, b) & \geq u(\kappa, f(\kappa))+u(f(\kappa), b)+b-\kappa \\
& =u(f(\kappa), b)+b-\kappa \\
& =f^{2}(\kappa)-b+b-\kappa \\
& =f(a \kappa)-\kappa \\
& =a+b(a \kappa-1)-\kappa=a-b
\end{aligned}
$$

where the fourth and fifth lines of (25) use the facts that $\kappa \leq 1$ and $a \kappa \geq 1$ respectively. Thus, $L(\kappa, b) \leq 0$, and so by Lemma $4, L(\kappa, b)=0$.

We claim that $\{k(t)\}$ is w-maximal. If the claim is false, there is some feasible path $\left\{k^{\prime}(t)\right\}$ from $\kappa$, a positive number, $\alpha$, and a positive integer $N$, such that for all $T>N$,

$$
\alpha \leq \sum_{t=0}^{T}\left[u\left(k^{\prime}(t), k^{\prime}(t+1)\right)-u(k(t), k(t+1))\right]
$$

For $T$ odd, write $T=2 S+1$, with $S$ a positive integer. Then:

$$
\begin{aligned}
\sum_{t=0}^{T} u\left(k^{\prime}(t), k^{\prime}(t+1)\right)= & \sum_{t=0}^{T} u\left(k^{\prime}(t), k^{\prime}(t+1)\right)+\sum_{s=0}^{S}\left[k^{\prime}(2 s+2)-k^{\prime}(2 s)\right] \\
& -\sum_{s=0}^{S}\left[k^{\prime}(2 s+2)-k^{\prime}(2 s)\right] \\
= & (S+1) \theta-\sum_{s=0}^{S} L\left(k^{\prime}(2 s), k^{\prime}(2 s+2)\right) \\
& -\left[k^{\prime}(T+1)-\kappa\right]
\end{aligned}
$$

and:

$$
\sum_{t=0}^{T} u(k(t), k(t+1))=(S+1) \theta-[k(T+1)-\kappa]
$$

since $L(k(2 s), k(2 s+2))=0$ for all $s \geq 0$. Combining (26), (27) and (28), one obtains:

$$
\alpha \leq\left[k(T+1)-k^{\prime}(T+1)\right]-\sum_{s=0}^{S} L\left(k^{\prime}(2 s), k^{\prime}(2 s+2)\right)
$$

Since $k(T+1)=b$ for all $T$ odd, $(29)$ implies that $k^{\prime}(T+1) \leq b-\alpha$ for all $T>N$, with $T$ odd. By Lemma 5 , we must then have for all $s$, satisfying 
$2 s>N+1$,

$$
\left.L\left(k^{\prime}(2 s), k^{\prime}(2 s+2)\right)\right) \geq \beta>0
$$

But, then, the right hand side of (29) becomes negative for large $T$, a contradiction.

Case (ii) $(\kappa \in[1, K])$

In this case, the consumption function $h$ generates the stock sequence $\{k(t)\}=(\kappa, b, 1, b, 1, \ldots)$ with associated consumption sequence $\{c(t+1)\}=$ $(f(\kappa)-b, 0, a-b, 0, a-b, \ldots)$.

For each $t=1,3,5, \ldots$, the value loss $L(k(t), k(t+2))=0$ by Lemma 4 .

We claim that $\{k(t)\}$ is $\mathrm{w}$-maximal. If the claim is false, there is some feasible path $\left\{k^{\prime}(t)\right\}$ from $\kappa$, a positive number, $\alpha$, and a positive integer $N$, such that for all $T>N$,

$$
\alpha \leq \sum_{t=0}^{T}\left[u\left(k^{\prime}(t), k^{\prime}(t+1)\right)-u(k(t), k(t+1))\right]
$$

For $T$ even, write $T=2 S$, with $S$ a positive integer. Then:

$$
\begin{aligned}
\sum_{t=1}^{T} u\left(k^{\prime}(t), k^{\prime}(t+1)\right)= & \sum_{t=1}^{T} u\left(k^{\prime}(t), k^{\prime}(t+1)\right)+\sum_{s=0}^{S-1}\left[k^{\prime}(2 s+3)-k^{\prime}(2 s+1)\right] \\
& -\sum_{s=0}^{S-1}\left[k^{\prime}(2 s+3)-k^{\prime}(2 s+1)\right] \\
= & S \theta-\sum_{s=0}^{S-1} L\left(k^{\prime}(2 s+1), k^{\prime}(2 s+3)\right) \\
& -\left[k^{\prime}(T+1)-k^{\prime}(1)\right]
\end{aligned}
$$

and:

$$
\sum_{t=1}^{T} u(k(t), k(t+1))=S \theta-[k(T+1)-k(1)]
$$

since $L(k(2 s+1), k(2 s+3))=0$ for $s \geq 0$. Also, since $f(\kappa)-b \geq f(1)-b=$ $a-b$, we have $u(k(0), k(1))=f(\kappa)-k(1)$, and:

$$
\begin{aligned}
u\left(k^{\prime}(0), k^{\prime}(1)\right)-u(k(0), k(1)) & \leq f(\kappa)-k^{\prime}(1)-f(\kappa)+k(1) \\
& =k(1)-k^{\prime}(1)
\end{aligned}
$$


Combining (31),(32),(33),(34), one obtains:

$$
\begin{aligned}
\alpha & \leq \sum_{t=0}^{T}\left[u\left(k^{\prime}(t), k^{\prime}(t+1)\right)-u(k(t), k(t+1))\right] \\
& \leq\left[k(T+1)-k^{\prime}(T+1)\right]-\sum_{s=0}^{S-1} L\left(k^{\prime}(2 s+1), k^{\prime}(2 s+3)\right)
\end{aligned}
$$

Since $k(T+1)=b$ for all $T$ even, (35) implies that $k^{\prime}(T+1) \leq b-\alpha$ for all $T>N$, with $T$ even. By Lemma 5 , we must then have for all $s$, satisfying $2 s>N$,

$$
\left.L\left(k^{\prime}(2 s+1), k^{\prime}(2 s+3)\right)\right) \geq \beta>0
$$

But, then, the right hand side of (35) becomes negative for large $T$, a contradiction.

Case (iii) $(\kappa \in[0, b))$

Since $f(0)=0$, the case in which $\kappa=0$ is trivial. Thus, we focus on the case in which $\kappa \in(0, b)$. Define the sequence $\{K(t)\}$ by $K(0)=\kappa$, and $K(t+1)=f(K(t))$ for $t \geq 0$. Then $\{K(t)\}$ is an increasing sequence, which converges to $K$. Consequently, we can find a smallest time period, $\tau$, such that $K(\tau) \geq b$; clearly, $\tau \geq 1$. The consumption function $h$ generates the stock sequence $\{k(t)\}=(\kappa, K(1), \ldots, K(\tau), K(\tau+1), b, 1, b, 1, \ldots)$ with associated consumption sequence $\{c(t+1)\}=(0, \ldots, 0, f(K(\tau+1))-b, 0, a-$ $b, 0, a-b, \ldots)$.

We claim that $\{k(t)\}$ is $\mathrm{w}$-maximal. If the claim is false, there is some feasible path $\left\{k^{\prime}(t)\right\}$ from $\kappa$, a positive number, $\alpha$, and a positive integer $N$, such that for all $T>N$,

$$
\alpha \leq \sum_{t=0}^{T}\left[u\left(k^{\prime}(t), k^{\prime}(t+1)\right)-u(k(t), k(t+1))\right]
$$

Consider the $T$, which can be written as $T=2 S+\tau+1$, with $S$ a positive 
integer. Then:

$$
\begin{aligned}
\sum_{t=\tau}^{T} u\left(k^{\prime}(t), k^{\prime}(t+1)\right)= & \sum_{t=\tau}^{T} u\left(k^{\prime}(t), k^{\prime}(t+1)\right)+\sum_{s=0}^{S}\left[k^{\prime}(2 s+\tau+2)-k^{\prime}(2 s+\tau)\right] \\
& -\sum_{s=0}^{S}\left[k^{\prime}(2 s+\tau+2)-k^{\prime}(2 s+\tau)\right] \\
= & (S+1) \theta-\sum_{s=0}^{S} L\left(k^{\prime}(2 s+\tau), k^{\prime}(2 s+\tau+2)\right) \\
& -\left[k^{\prime}(T+1)-k^{\prime}(\tau)\right]
\end{aligned}
$$

and:

$$
\sum_{t=\tau}^{T} u(k(t), k(t+1))=(S+1) \theta-[k(T+1)-k(\tau)]
$$

since $L(k(2 s+\tau), k(2 s+\tau+2))=0$ for all $s \geq 0$ (for the reason given in Case (i) above). Combining (38) and (39), one obtains:

$$
\begin{gathered}
{\left[\sum_{t=\tau}^{T} u\left(k^{\prime}(t), k^{\prime}(t+1)\right)-\sum_{t=\tau}^{T} u(k(t), k(t+1))\right]=\left[k^{\prime}(\tau)-k(\tau)\right]+} \\
\left.\left[k(T+1)-k^{\prime}(T+1)\right]-\sum_{s=0}^{S} L\left(k^{\prime}(2 s+\tau), k^{\prime}(2 s+\tau+2)\right)\right)
\end{gathered}
$$

We now show that:

$$
\left[\sum_{t=0}^{\tau-1} u\left(k^{\prime}(t), k^{\prime}(t+1)\right)-\sum_{t=0}^{\tau-1} u(k(t), k(t+1))\right] \leq\left[k(\tau)-k^{\prime}(\tau)\right]
$$

Note that $k(t+1)=f(k(t))$ for $t=0, \ldots, \tau-1$, and so:

$$
\sum_{t=0}^{\tau-1} u(k(t), k(t+1))=0
$$

Note that $k^{\prime}(t) \leq k(t)<b$ for $t=0, \ldots, \tau-1$, so $\left[f\left(k^{\prime}(t)\right)-k^{\prime}(t)\right] \leq[f(k(t))-$ 
$k(t)]$ for $t=0, \ldots, \tau-1$. Consequently,

$$
\begin{aligned}
\sum_{t=0}^{\tau-1} u\left(k^{\prime}(t), k^{\prime}(t+1)\right) & \leq \sum_{t=0}^{\tau-1}\left[f\left(k^{\prime}(t)\right)-k^{\prime}(t+1)\right] \\
& =\sum_{t=0}^{\tau-1}\left[f\left(k^{\prime}(t)\right)-k^{\prime}(t)\right]+\left[\kappa-k^{\prime}(\tau)\right] \\
& \leq \sum_{t=0}^{\tau-1}[f(k(t))-k(t)]+\left[\kappa-k^{\prime}(\tau)\right] \\
& =\sum_{t=0}^{\tau-1}[f(k(t))-k(t+1)]-[\kappa-k(\tau)]+\left[\kappa-k^{\prime}(\tau)\right] \\
& =\left[k(\tau)-k^{\prime}(\tau)\right]
\end{aligned}
$$

Clearly, (41) follows from (42) and (43).

Combining (37),(40) and (41), we obtain:

$$
\begin{aligned}
\alpha \leq & \sum_{t=0}^{T}\left[u\left(k^{\prime}(t), k^{\prime}(t+1)\right)-u(k(t), k(t+1))\right] \\
\leq & {\left[k(T+1)-k^{\prime}(T+1)\right]-} \\
& \left.\sum_{s=0}^{S} L\left(k^{\prime}(2 s+\tau), k^{\prime}(2 s+\tau+2)\right)\right)
\end{aligned}
$$

Since $k(T+1)=b$ for all $T$ of the form $T=2 S+\tau+1$, (44) implies that $k^{\prime}(T+1) \leq b-\alpha$ for all $T>N$, with $T$ of the form $T=2 S+\tau+1$. By Lemma 5, we must then have for all $s$, satisfying $2 s+\tau+2>N+1$,

$$
\left.L\left(k^{\prime}(2 s+\tau+2), k^{\prime}(2 s+\tau+4)\right)\right) \geq \beta>0
$$

But, then, the right hand side of (44) becomes negative for large $T$, a contradiction.

This completes the proof of (a), and therefore of (A). The proof of (B) is straightforward, since the dynamical system is given by (DS). 


\section{References}

[1] Asheim, G.B., W. Bossert, Y. Sprumont and K. Suzumura, InfiniteHorizon Choice Functions, Economic Theory 43 (2010) 1-21.

[2] Asheim, G.B., W.Buchholz and B. Tungodden, Justifying Sustainability, J.Environmental Economics and Management 41 (2001), 252-268.

[3] d'Aspremont, C., Axioms for Social Welfare Orderings, in L. Hurwicz, D. Schmeidler and H. Sonnenschein (eds.), Social Goals and Social Organizations: Essays in Memory of Elisha Pazner, Cambridge University Press, Cambridge, (1985), 19-76.

[4] Atsumi, H., Neoclassical growth and the efficient program of capital accumulation, Rev. Econ. Stud. 32 (1965) 127-136.

[5] Basu, K. and T. Mitra, Utilitarianism for infinite utility streams: A new welfare criterion and its axiomatic characterization, J. Econ. Theory 133 (2007) 350-373.

[6] Brock, W.A., On existence of weakly maximal programmes in a multisector economy, Rev. Econ. Stud. 37 (1970) 275-280.

[7] Gale, D.: On Optimal Development in a Multi-Sector Economy, Review of Economic Studies 34 (1967), 1-18.

[8] Hardy, G.H., J.E. Littlewood and G. Polya, Inequalities, Second Edition, Cambridge University Press, Cambridge, 1952.

[9] McKenzie, L.W., Accumulation programs of maximum utility and the von Neumann facet, Value, Capital and Growth, ed. J.N. Wolfe, Edinburgh University Press, (1968), 353-383.

[10] Radner, R., Paths of economic growth that are optimal with regard only to final states: A turnpike theorem, Rev. Econ. Stud. 28 (1961), 98-104.

[11] Sen, A.K.: Collective Choice and Social Welfare; Edinburgh, Oliver\&Boyd, 1971.

[12] Suppes, P.: Some Formal Models of Grading Principles, Synthese 6 (1966), 284-306.

[13] Svensson, L.-G.: Equity among Generations, Econometrica 48 (1980), 1251-1256. 